\title{
Le voyage discret des plantes
}

Abelmoschus moschatus (Malvaceae) et Zingiber zerumbet (Zingiberaceae) en Amérique tropicale

The discret journey of plants: Abelmoschus moschatus (Malvaceae) and Zingiber zerumbet (Zingiberaceae) in the Neotropics

Pierre Grenand, Marie-Françoise Prévost et Marie Fleury

\section{OpenEdition}

\section{Journals}

Édition électronique

URL : http://journals.openedition.org/ethnoecologie/733

DOI : 10.4000/ethnoecologie.733

ISSN : 2267-2419

Éditeur

Laboratoire Eco-anthropologie et Ethnobiologie

Référence électronique

Pierre Grenand, Marie-Françoise Prévost et Marie Fleury, « Le voyage discret des plantes », Revue d'ethnoécologie [En ligne], 1 | 2012, mis en ligne le 30 juin 2012, consulté le 30 avril 2019. URL : http:// journals.openedition.org/ethnoecologie/733 ; DOI : 10.4000/ethnoecologie.733

Ce document a été généré automatiquement le 30 avril 2019.

Revue d'ethnoécologie est mis à disposition selon les termes de la licence Creative Commons Attribution - Pas d'Utilisation Commerciale - Pas de Modification 4.0 International. 


\title{
Le voyage discret des plantes
}

\author{
Abelmoschus moschatus (Malvaceae) et Zingiber zerumbet \\ (Zingiberaceae) en Amérique tropicale \\ The discret journey of plants: Abelmoschus moschatus (Malvaceae) and \\ Zingiber zerumbet (Zingiberaceae) in the Neotropics
}

Pierre Grenand, Marie-Françoise Prévost et Marie Fleury

\section{Introduction}

1 Au sein du débat foisonnant sur la notion de propriété intellectuelle, Bennett et Prance (2000) ont tenté, dans un effort salutaire de relativisation des flux de gènes et de savoir, d'attirer l'attention sur l'introduction des plantes médicinales dans le nord de l'Amérique du Sud. Ce ne sont pas moins de deux cent seize plantes introduites de l'Ancien Monde qui sont aujourd'hui utilisées par les communautés rurales et autochtones de cette région.

2 Cet article suggère que les cheminements de diffusion et d'emprunts de l'Ancien vers le Nouveau Monde sont loin d'avoir été étudiés, à l'exception d'espèces d'importance économique reconnue.

3 Les deux exemples que nous développons ici, Abelmoschus moschatus Medik. et Zingiber zerumbet (L.) Sm., relèvent de cette démarche d'élucidation et leur choix a été dicté par la difficulté que nous avons eue à les reconnaître comme plantes introduites.

\section{Les espèces : cadrage botanique}

4 Abelmoschus moschatus est un sous-arbrisseau de un à deux mètres de haut, parfois plus, appartenant à un genre de Malvacées comprenant six espèces natives de l'Asie tropicale, de l'Insulinde et des îles du Pacifique. Ce genre est très proche du genre Hibiscus. Les feuilles sont lobées, les fleurs jaunes. Le fruit est une capsule fusiforme $(5 \times 3 \mathrm{~cm})$, anguleuse, poilue, contenant de nombreuses graines ovoïdes-réniformes noires (Bates 
1968 : 103-106). Il s'agit d'une espèce bien individualisée qui n'est jamais confondue avec les espèces alimentaires proches, A. esculentus (L.) Moench et A. manihot (L.) Medik.

Photo 1. Capsules sèches d'Abelmoschus moschatus ; c'est à ce stade que les graines parfumées sont utilisées

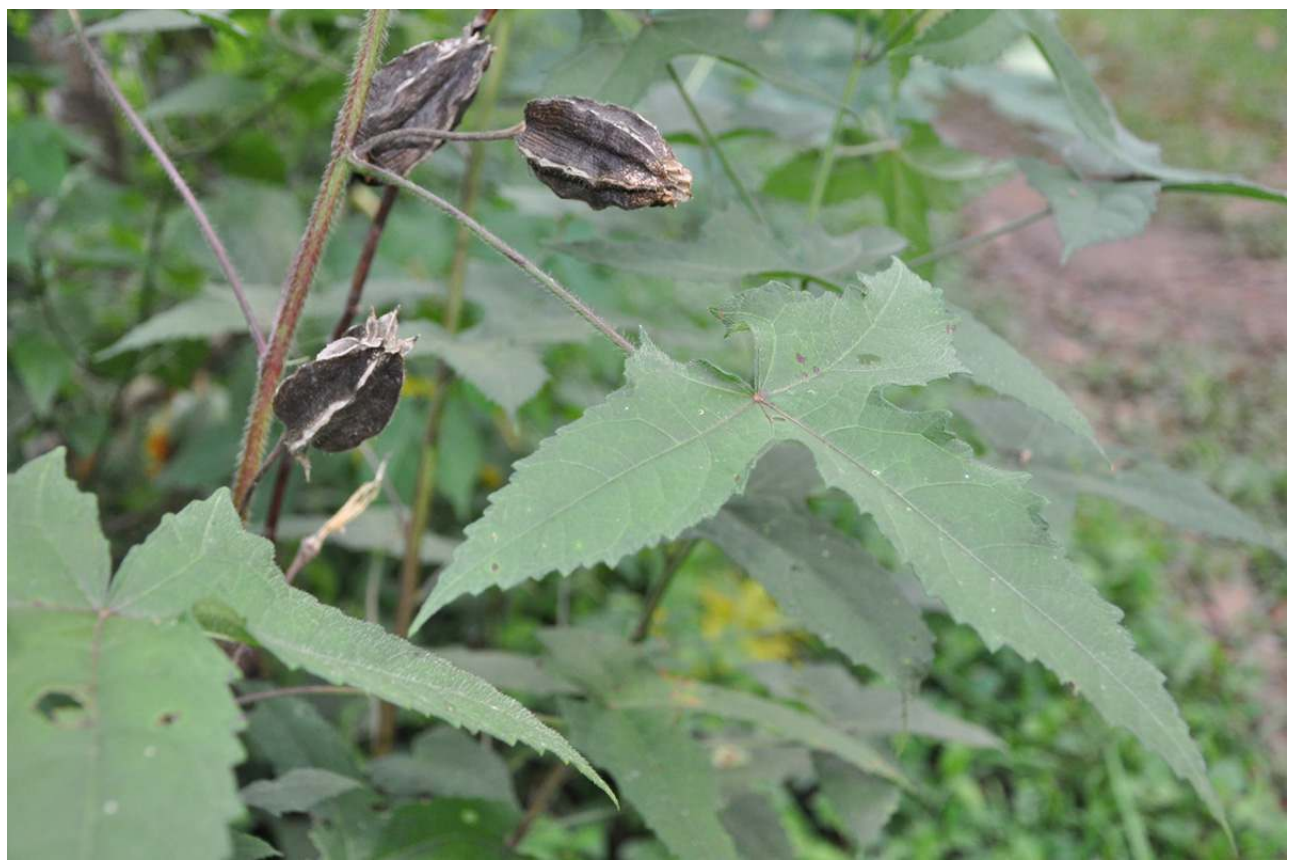

Cliché M. Fleury

Photo 2. Fleur d'Abelmoschus moschatus

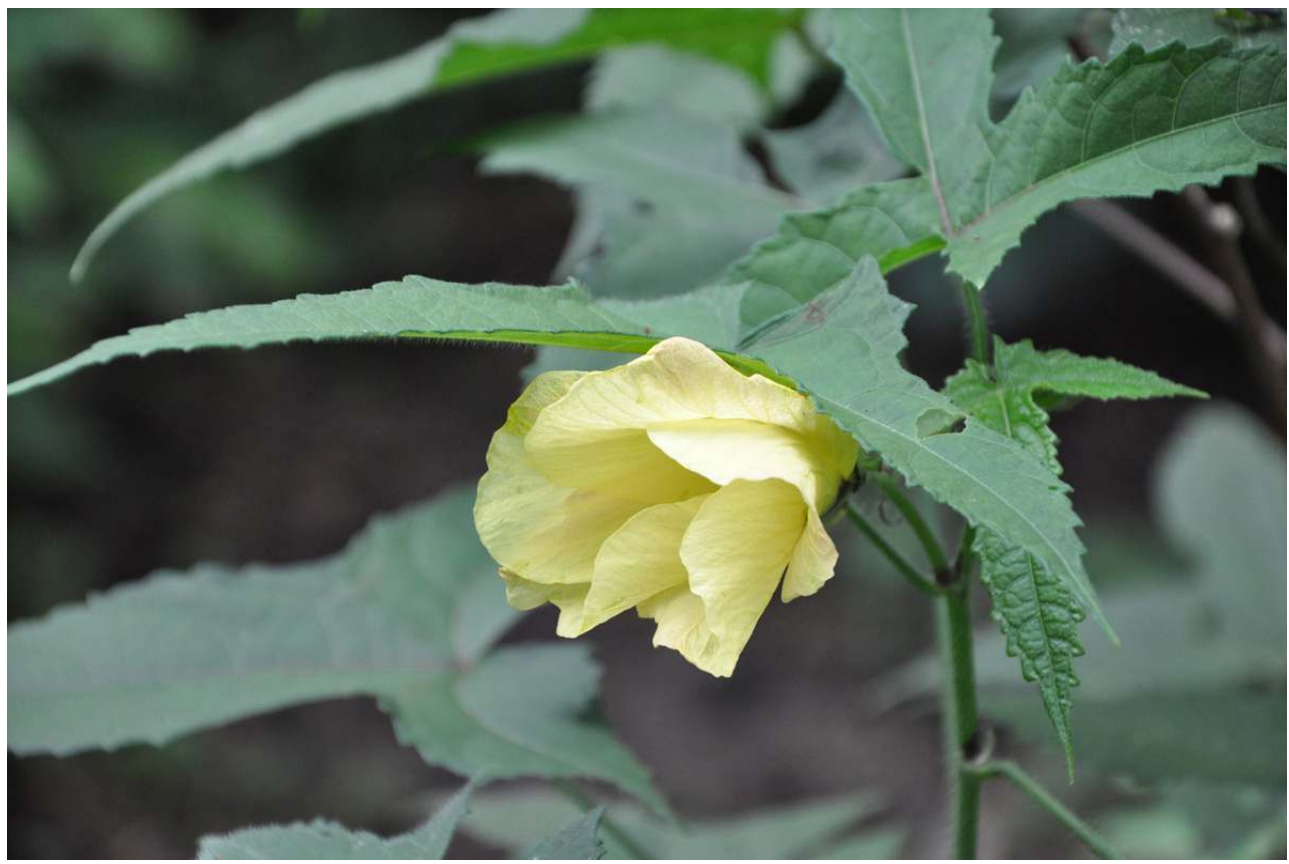

Cliché M. Fleury 
Photo 3. Inflorescence de Zingiber zerumbet

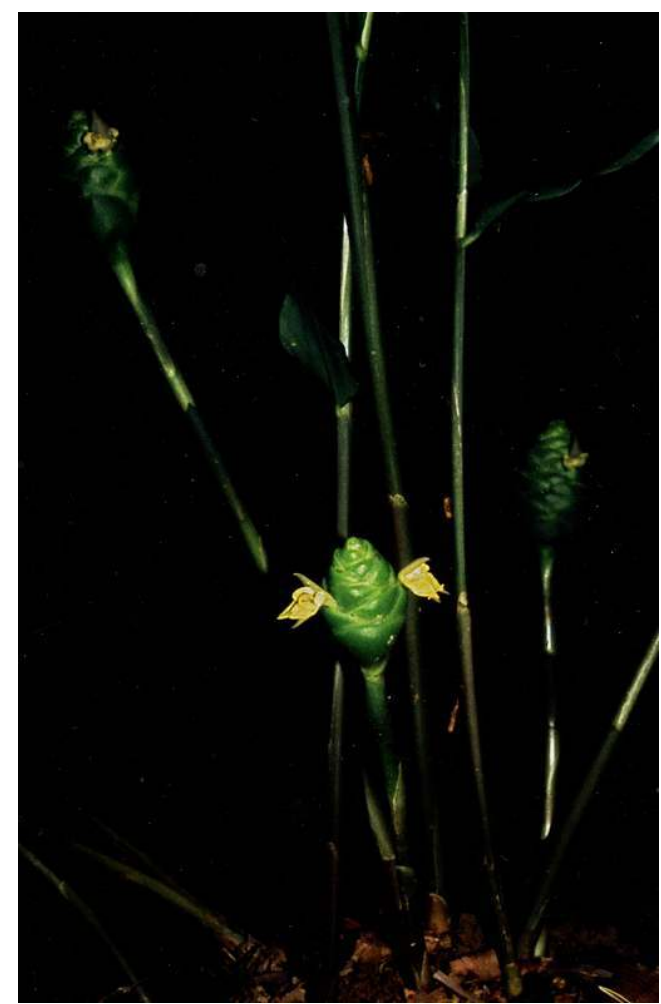

Cliché M.F. Prévost

Zingiber zerumbet appartient à un genre très important de Zingibéracées comportant une centaine d'espèces toutes originaires d'Asie tropicale. C'est une plante herbacée vigoureuse pouvant atteindre deux mètres de haut. Comme chez toutes les espèces $\mathrm{du}$ genre, les rejets présentent de nombreuses feuilles alternes, sessiles et lancéolées. Les inflorescences comprimées, ayant l'aspect d'une petite pomme de pin, naissent séparément de la tige feuillée, portées par des pédoncules charnus d'une cinquantaine de centimètres; les fleurs jaune pâle sont protégées par des bractées verdâtres qui constituent l'essentiel visible de l'inflorescence (Burtt \& Smith 1983: 492, 495). Le rhizome tubéreux est ramifié et la pulpe aromatique varie du blanc pâle au jaune vif.

\section{Histoire d'une (re)découverte}

Entre 1971 et 1976, l'un d'entre nous (P. Grenand) a partagé au quotidien la vie de trois communautés wayãpi, installées dans le haut Oyapock. En parallèle à un programme d'éducation bilingue, furent menées tout au long de ces années des enquêtes ethnographiques en profondeur.

7 C'est en 1974 que fut collecté Zingiber zerumbet au village wayãpi de Zidock, alors qu' Abelmoschus moschatus avait déjà été observé et dessiné sur le rio Kouc en 1969, mais ce n'est qu'en 1978 pour la première espèce et dans les années 1980 pour la seconde qu'elles furent définitivement identifiées. Pour les Wayãpi il s'agissait d'espèces ancestrales qu'ils considéraient avoir transportées à travers leurs migrations de l'Amazone à la Guyane au cours des XVIII et XIX ${ }^{e}$ siècles. 
Zingiber zerumbet, connu principalement sous le nom de yamalatay, était présent en petite quantité et sa localisation en végétation secondaire nous laissait supposer qu'il s'agissait d'une plante spontanée adaptée à ce genre de milieu.

9 Abelmoschus moschatus, bien que très connu sous le nom de moypoã, était considéré comme perdu à Trois Sauts, lors de nos premières enquêtes. En 1975, suite à l'ouverture d'un nouvel abattis en amont de Trois Sauts sur un site d'ancien village ${ }^{1}$, les Wayãpi du village du chef Roger indiquèrent l'invasion d'une partie de la parcelle par le moypoã. C'était un exemple particulièrement intéressant de résilience des graines. À partir de ce stock, la plante fut partagée entre l'ensemble des communautés du haut Oyapock.

Ce n'est que lors de leur identification que nous comprîmes qu'il s'agissait d'une espèce introduite. Cette résolution de l'énigme entraîne, de fait, d'autres questions, dont les réponses, que nous allons essayer de formuler et d'étayer au cours d'un long voyage, ne sont bien souvent que des suppositions.

\section{Sur la piste...}

\section{Abelmoschus moschatus et Zingiber zerumbet dans l'Ancien Monde et le Pacifique}

Abelmoschus moschatus est une espèce clairement individualisée, bien reconnue partout où elle existe et dont le cheminement peut être aisément suivi de continent en continent. Il n'en va pas de même de Zingiber zerumbet dont la confusion avec d'autres Zingibéracées et en tout premier lieu Zingiber officinale Roscoe est souvent faite; hors de leur terre d'origine, les usages précis de $Z$. zerumbet sont aussi plus délicats à cerner et semblent souvent recouvrir Z. officinale et, plus rarement, Curcuma longa L. En outre, de nombreuses sources précisent le caractère spontané, tant d'Abelmoschus moschatus que des Zingiber, dans les régions néotropicales, au moins dans des conditions écologiques favorables telles que friches, végétation secondaire, savanes...

\section{Abelmoschus moschatus}

L'espèce, connue en français sous le nom d'ambrette, est signalée de l'Inde, de la Chine du Sud, du Sud-Est asiatique, de l'Indonésie, de Nouvelle-Guinée, du nord de l'Australie et des îles du Pacifique. Elle est aussi connue d'Afrique de l'Ouest où elle a été introduite.

En Inde, ses nombreux usages en médecine ayurvédique attestent de sa présence très ancienne : les graines aromatiques sont utilisées comme tonique et considérées comme rafraîchissantes, ophtalmiques, cardiotoniques, stomachiques, constipantes, carminatives, pectorales, diurétiques, stimulantes, antispasmodiques, déodorantes et efficaces contre les excès des éléments kapha et vata, les douleurs intestinales et les stomatites. Elles soulagent la soif et calment les vomissements (Agharkar 1991). Différentes parties de la plante sont aussi utilisées comme remède pour soigner les crampes, la mauvaise circulation, les douleurs articulaires et dans le traitement de la dépression et de l'anxiété. L'usage des graines comme aphrodisiaque est également signalé (Bown 1995) ainsi que pour soigner les morsures de serpent (Lindley 1985).

Elle ne semble pas avoir été anciennement cultivée en Europe, bien que son usage aromatique y fût connu (www.eFloras.org). 
15 En effet, les graines distillées donnent une huile essentielle, appelée par les parfumeurs «absolue de graines d'ambrette». Actuellement cette essence est interdite en Europe à cause de la présence de musc ambrette, à propriété allergisante. On la synthétise maintenant pour l'industrie du parfum. Son odeur est musquée, animale et légèrement fruitée. Elle entre ainsi dans la composition de plusieurs parfums prestigieux ( www.auparfum.com).

16 Autrefois, ses graines à odeur d'ambre gris servaient à parfumer la poudre pour les cheveux (www.patrimoine-de-france.org/). Actuellement, on trouve en vente sur internet de l'extrait $\mathrm{CO}_{2}$ d'ambrette ${ }^{2}$ issue du Pérou, comme substitut de l'huile essentielle pour la fabrication de parfums. On la conseille surtout pour la préparation de parfums et aprèsrasages masculins, à cause de son effet hormon like, c'est-à-dire simulant les hormones. Décrite comme une fragrance séductrice, elle est également utilisée pour la préparation de boissons et de cocktails (www.aroma-zone.com).

Abelmoschus esculentus).

\section{Zingiber}

Zingiber officinale et $Z$. zerumbet sont deux espèces bien connues de l'Asie tropicale. Ainsi, selon la Flore de Ceylan (Burtt \& Smith 1983 : 495, 498), Z. officinale est cultivé tandis que $Z$. zerumbet, essentiellement médicinal en raison de son amertume, est cultivé mais aussi naturalisé. Cette dernière espèce serait originaire de l'Inde.

Dans l'Ancien Monde, les sources consultées évoquent le plus souvent le gingembre, Zingiber officinale, comme plante anciennement cultivée déjà mentionnée par Confucius (551-479 av. J.-C.) et dont l'introduction en Asie Mineure daterait de l'époque de l'occupation romaine; la première occurrence en Europe occidentale remonte à 1292 (Rosengarten 1969 ; Smith 1976 : 324). Une autre source indique que, dès 1400 av. J.C., les Phéniciens en faisaient commerce en Grèce. C'était une épice très recherchée en Europe pendant le Moyen Âge (Longuefosse 1995 : 96).

20 Vers l'est, le voyage de Zingiber zerumbet est sans doute plus ancien que celui de $Z$. officinale, puisqu'il est considéré comme « une ancienne plante mélanésienne » introduite lors des migrations anciennes, soit il y a plus de dix millénaires, alors que le gingembre commun (Z. officinale) ne serait parvenu en Mélanésie qu'au XIX ${ }^{\mathrm{e}}$ siècle (Walter \& Lebot 2003 : 296-297).

21 Actuellement il est cultivé aux Philippines, au Sri Lanka, en Inde, en Thaïlande ( www.toildepices.com), en Indonésie, à Brunei, en Indochine et à Hawaï où il est, de nos jours, une plante médicinale importante. Ses rhizomes servent à soigner l'indigestion et les douleurs, les rages de dents et les entorses (http://en.wikipedia.org/wiki/ Zingiber_zerumbet).

22 En Polynésie comme à Hawaï, on le nomme shampoo ginger en raison de son utilisation cosmétique: on extrait le lait des inflorescences en les roulant entre les mains pour l'appliquer sur les cheveux et sur le corps. Ce «lait» est utilisé dans certains shampooings du commerce pour renforcer la brillance des cheveux et les adoucir. Le rhizome peut être employé comme condiment et les feuilles (jeunes pousses) comme légume. En Nouvelle-Guinée, la plante est utilisée dans les rituels initiatiques des hommes et rendrait les femmes stériles en cas d'utilisation... (www.toildepices.com). 
Parallèlement,

«Le gingembre (Zingiber officinale) est la panacée de la médecine asiatique; il soigne quasiment tous les maux [...: il est] apéritif (stimule la sécrétion salivaire et celle des sucs gastriques ainsi que les mouvements intestinaux), stomachique, carminatif, digestif (facilite la digestion, réchauffe l'estomac: des lanières macérées dans du jus de lime sont un digestif birman), antibactérien, antiseptique, antimicrobien, nématocide, molluscicide, antischistosomal, stimulant, fortifiant, réchauffant [...] fébrifuge [... : Il] soulage les problèmes circulatoires, baisse le taux de cholestérol; en infusion [il est bu] contre la toux, la grippe, et les maux de gorges divers. [Il est aussi] antiémétique, calme le mal des transports et de mer. [Enfin on peut l'utiliser] contre le mal de tête et contre les rhumatismes » ( www.toildepices.com).

\section{Les deux plantes en Amérique}

Les deux espèces offrent un contraste intéressant car nous allons voir que si la première est bien connue, la seconde l'est beaucoup moins et de surcroît largement confondue avec Zingiber officinale. Pour cette raison, nous indiquons les citations concernant cette espèce parallèlement à celles concernant $Z$. zerumbet.

\section{Abelmoschus moschatus}

Une extraction du Virtual Herbarium ${ }^{3}$ indique dix occurrences pour l'Amérique tropicale avec comme lieu de collecte le Belize, le Costa Rica, la Guyane française, les Antilles néerlandaises, le Pérou, Porto Rico et le Brésil. Une autre extraction de l'herbier du Missouri Botanical Garden ${ }^{4}$ donne cent onze occurrences et nous permet d'ajouter aux pays précédents, le Mexique, le Honduras, le Nicaragua, le Panama, la Colombie, l'Équateur et le Venezuela. Sur cette base, on peut avancer que l'espèce est présente à peu près partout en Amérique tropicale avec une représentation particulièrement importante dans la zone circum-caraïbe. Cette réalité contemporaine est à relier sans aucun doute avec une introduction ancienne.

Marcgrave ([1648] 1942: 45) nous livre, sous le nom d'Alcaea hirsuta, une description détaillée de l'espèce, ainsi qu'une illustration ${ }^{5}$. Il nous la décrit comme cultivée, insiste sur la beauté de la fleur, indique le goût musqué des graines mâchées mais ne fournit ni usage, ni nom vernaculaire. Si l'on excepte les sources concernant la Guyane (cf. infra), il nous faut attendre Grisebach ([1864] 1963: 84) qui indique "naturalized in Jamaica, the Caribbean Islands; [and in all tropical countries; said to be spontaneous in Guiana!, and Central America!] ».

En 1897, Duss ([1897] 1972 I : 73-74) précise que l'espèce est naturalisée et cultivée en Martinique et Guadeloupe. Selon cet auteur: "Descourtilz [qui écrit au début du xix ${ }^{e}$ siècle] range cette plante dans la classe des antispasmodiques aromatiques et en vante les propriétés médicinales. Dans le pays elle n'est pas employée souvent ».

Selon Matta (1938: 45) elle est originaire de l'Inde mais connue en Amazonie brésilienne ; les graines triturées exhalent un parfum de musc et fournissent une huile essentielle.

Plus récemment, on trouve des usages concernant les communautés métisses ou indigènes. Au Veracruz (Mexique), A. moschatus est cultivé et naturalisé en végétation secondaire et même ornementale ; les graines sont un substitut du café ; mastiquées, elles sont utilisées comme remède pour les morsures de serpents venimeux (Fryxell 1992: 16).

Revue d'ethnoécologie, 1 | 2012 
30 En Amazonie péruvienne, c'est un «cultivated ornamental at Yanomono. Seeds used to make necklaces for children suffering bronchitis and cough ... Seeds used as tonic and antispasmodic». (Duke \& Vasquez 1994:13).

31 Enfin, selon Schultes \& Raffauf (1990: 289), " the Siona are reported to value this plant as a remedy for snake bite. It has several medicinal uses amongst the Tikunas ». Ainsi elle est un remède contre la diarrhée (feuilles + quelques feuilles d'Anacardium occidentale); les graines écrasées dans un bouillon de poule soignent la bronchite; la décoction de feuilles est bonne contre les douleurs menstruelles ou les règles excessives ; enfin, la décoction longue bue à raison d'une tasse par jour aide le travail des parturientes.

\section{Zingiber}

Zingiber officinale est infiniment plus présent dans les sources que Zingiber zerumbet en Amérique tropicale.

Une extraction du Virtual Herbarium du New York Botanical Garden n'indique que Porto Rico comme lieu de collecte de Zingiber zerumbet avec douze occurrences pour l'Amérique tropicale.

Marcgrave ([1648] 1942: 17-18) fournit pourtant l'une des plus anciennes mentions (illustrée de surcroît) des gingembres en Amérique qu'il nomme Zinziber. Il évoque deux espèces, une " mâle » et une "femelle ", en s'appuyant sur F. Ximenes. La plante mâle est plus robuste, avec une racine amère et des usages médicinaux (douleurs d'estomac, contre-poison et soins des blessures d'origine vénéneuse). La plante femelle est selon lui le gingembre commun qui sert avant tout de condiment et d'aliment de dessert. Il nous semble vraisemblable qu'il s'agisse bien des deux espèces $Z$. zerumbet et $Z$. officinale. 
Figure 1. Représentation probable d'Abelmoschus moschatus

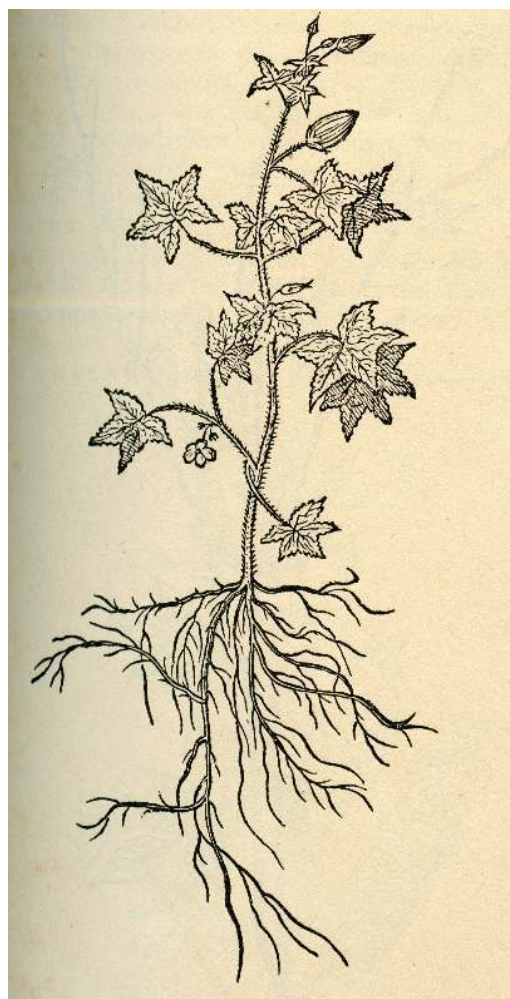

Marcgrave (1648) Histoire Naturelle du Brésil II : 583).

41 Selon Longuefosse (1995: 97), on l'emploie aux Antilles françaises pour les affections digestives, le mal de transport, les douleurs dentaires, les états grippaux et la toux ; il est aussi tonique, aphrodisiaque, antirhumatismal. 
$42 \mathrm{Au}$ Pérou, l'espèce est cultivée. «Macerated rhizomes in aguardiente for arthritis; believed to invigorate males. Rhizome decoction used for diarrhea, and, with a pinch of cinnamon, stomachaches. Also used as an antiflatulent and spice » (Duke \& Vasquez 1994 : 181).

Chez les populations amérindiennes d'Amazonie, les observations d'usages de Zingiber officinale sont contemporaines, mais ces emplois semblent si bien installés qu'ils ne peuvent être récents. En voici une revue:

"Many Indians of the Northwest Amazon employ the rhizome, grated into water, slightly boiled and taken internally as a remedy for stomach ache and diarrhea. Amongst the Tikunas, the entire plant is crushed and boiled in water to make a decoction considered to be efficacious in curing bodily pains" (Schultes \& Raffauf 1990: 473).

"Reports from the Shuar [Stirling 1938], the Aguaruna [Brown 1981], an Achuar [Descola 1988] mentioned the use of ajej (Zingiber officinale Roscoe) as a hallucinogen, but this species has no known hallucinogenic principals. The Cariña apply a mixture of tobacco and Z. officinale to the eyes of apprentice shamans so that they can see spirits [Wilbert 1987]. Extracts from ginger have a depressant effect on the central nervous system [Schultes \& Raffauf 1990] and large doses may be hallucinogenic [Lewis \& Elvin-Lewis 1977]. Although it is one of their most important medicinal plants [Bennett et al. in press], I found no hallucinogenic uses of ginger among the Shuar." (Bennett 1992 : 490)

Les Urubu-Kaapor utilisent Z. officinale pour soigner la grippe et les maux de gorge (Balée 1994 : 309). Enfin, les Tacana l'utilisent pour atténuer les spasmes intestinaux et les rhumatismes, soigner les dysenteries, et soulager les brûlures buccales liées à la consommation de la coca (Anonyme 1999 : 329).

De ce qui précède, il ressort que Zingiber officinale est infiniment mieux connu que Zingiber zerumbet en Amérique tropicale. Néanmoins il n'est pas exclu que certaines occurrences de la littérature recouvrent autant l'une que l'autre espèce.

\section{Les deux plantes dans les Guyanes}

\section{Dans la littérature}

\section{Abelmoschus moschatus}

La première mention d'A. moschatus provient de la flore de Fusée Aublet ([1775] 1977 : 707) : « Hibiscus abelmoschus ... l'ambrette, ou graine musquée. Cette plante est cultivée dans la Guiane, ainsi qu'à l'Isle de France ».

Plus tard, Heckel (1897 : 84-85) précise : « Ambrette ; calalou musqué. Hibiscus abelmoschus L. (Malvacées). Les graines à odeur de musc sont employées comme masticatoire contre la carie dentaire et l'haleine fétide. Leur infusion est considérée comme céphalique et stomachique ; on la regarde aussi comme antispasmodique ».

Ostendorf (1962 : 121) indique, sans détails, la culture de cette plante au Surinam pour ses graines à odeur musquée.

Nous trouvons une brève mention de cette espèce comme plante magique en Guyana dans Van Andel (2000 II : 298). 


\section{Zingiber}

\section{La plus ancienne
$(1741: 118-119)$.}

«Cette Plante vient si aisément à Cayenne, qu'elle semble être naturelle au Pays : les Indiens ont soin d'en avoir toujours quelques pieds dans leurs Plantations; ils s'en servent dans la toux opiniâtre \& les rhûmes invétérés, pour faciliter le crachement ».

51

$$
\begin{aligned}
& \text { L'affirmation que cette plante est déjà fermement adoptée par les autochtones mérite } \\
& \text { d'être soulignée. }
\end{aligned}
$$
Il indique aussi qu'il donne de l'appétit aux convalescents et est utilisé comme condiment.
«Amomum (Zingiber) [...] Le gingembre est cultivé sur différentes habitations de l'île de Caïenne \& de la Guiane. On confit les racines. Il se cultive aussi à l'île de France » (Fusée Aublet $1775: 2$ ).

De ces deux citations, on ne peut déduire s'il s'agit d'une ou de deux espèces.

Heckel (1897 : 115) ne signale que : « Gingembre, Amomum zingiber L.; Zingiber officinale Rosc. (Scitaminées). Stomachique, stimulant, aromatique. Introduit de l'Inde tropicale ».

Bière de gingembre et huile essentielle sont indiquées selon Lemée (1956: 21) pour la Guyane française.

Zingiber cassumunar Roxb., Z. officinale Roscoe et Z. zerumbet (L.) Sm. sont plus récemment signalés au Surinam comme d'origine asiatique (Maas 1985 : 62-63). Le premier est un remède contre les maux d'estomac et les crampes, le second sert à préparer du ginger-ale et des ginger-cookies, le troisième est utilisé pour assaisonner le riz (dans la communauté javanaise) et comme plante médicinale, sans autre précision.

Van Andel (2000 II : 298), dans son travail sur l'ethnobotanique du nord-ouest de la Guyana, signale l'usage de Zingiber officinale alors qu'elle évoque Z. zerumbet uniquement comme plante ornementale. Zingiber officinale est prescrit en thé associé à Pothomorphe peltata (Pipéracées) pour nettoyer la matrice après l'accouchement (ibid. I : 274 et II : 177). Chez les Arawak, il sert à soigner la malaria et les hémorragies.

57 Dans l'ensemble des sources consultées pour l'Amérique tropicale et les Guyanes, Abelmoschus moschatus est bien présent depuis le $\mathrm{XVII}^{\mathrm{e}}$ siècle, mais les mentions postérieures sont plutôt maigres avant le $\mathrm{xx}^{\mathrm{e}}$ siècle.

Pour ce qui est de Zingiber zerumbet, il est absent des sources anciennes - si l'on excepte la mention discutable de Marcgrave - alors que Z. officinale est présent au moins depuis le $\mathrm{XVII}^{\mathrm{e}}$ siècle. Ce silence des sources ne signifie pas pour autant une absence sur le terrain, car c'est surtout la floraison qui permet de les distinguer.

\section{Nos observations}

9 Pour les trois espèces, les échantillons ont été relevés dans un contexte anthropique ${ }^{6}$ et la presque totalité mentionne un nom vernaculaire.

Dans l'Herbier de Guyane (CAY), il existe onze collectes d'Abelmoschus moschatus toutes liées, sauf une, à la présence d'une communauté humaine. Cette plante est connue de toutes les populations guyanaises (cf. Annexe).

Dans le même herbier, il existe cinq collectes de Zingiber officinale et sept de Zingiber zerumbet. Ces deux espèces sont également connues de toutes les populations guyanaises 
(cf. Annexe) et semblent être bien distinguées, sauf pour Zingiber zerumbet qui est parfois associé avec Curcuma longa par les Créoles.

\section{Abelmoschus moschatus}

Abelmoschus moschatus trouve actuellement de nombreux usages en Guyane où on l'utilise avant tout pour soigner les morsures de serpent venimeux, les propriétés stimulantes et antispasmodiques des graines étant connues depuis longtemps (Lemée 1956) tant en Asie qu'en Amérique. Les Créoles absorbent six graines par jour pendant cinq jours, tandis que les Wayãpi se contentent d'une vingtaine de graines fraîches ou sèches en une seule prise. Cet usage a également été indiqué chez les Aluku et les Wayana où elle est appelée ëkëi epit, «le remède du serpent ». Les Créoles attribuent par ailleurs à d'autres parties de la plante des propriétés fébrifuges, antalgiques et anti-inflammatoires. Ainsi le broyat gluant des feuilles est appliqué en cataplasme sur le front contre les céphalées. La racine fraîche, écrasée et mêlée à du suif est appliquée en onguent sur les piqûres envenimées de clous ou d'épines. Enfin, la macération des racines dans le rhum est utilisée en friction contre la fièvre.

Chez les Palikur, la décoction des feuilles et des capsules vertes est utilisée, comme Abelmoschus esculentus, en bain pour favoriser l'accouchement, usage que l'on a déjà trouvé chez les Tikuna d'Amazonie péruvienne (Duke \& Vasquez 1994).

Chez les Aluku, l'espèce est plantée autour de l'abattis pour empêcher les cabiais ( Hydrochaeris hydrochaeris) de venir manger les plantes cultivées; mais l'usage du sabre pour la couper est proscrit sous peine de voir l'effet s'annuler (Fleury \& Topo à paraître). Enfin, Palikur et Créoles signalent l'utilisation de cette plante à des fins de sorcellerie. Chez les Palikur en particulier, elle sert à neutraliser les “poisons" préparés avec les Caladium (Aracées).

\section{Zingiber}

Zingiber zerumbet est surtout utilisé chez les Wayãpi, où l'espèce est intégrée dans la culture et le souvenir de son introduction totalement gommé de la mémoire collective. Les racines sont avant tout considérées comme un charme protecteur contre les atteintes de l'anaconda (Eunectes murinus) dont la plante est une émanation. Elle tient de ce rapport éminent son deuxième nom, moyupoã, "remède de l'anaconda ». Elle est utilisée tout particulièrement pendant la danse de l'anaconda (moyu tule) ${ }^{7}$. Lors de l'ouverture de cette danse, les danseurs tournent autour d'une calebasse pleine de macération de racine écrasée de Zingiber zerumbet (Beaudet 1997 : 139). Ce liquide est ensuite appliqué à l'aide d'une plume caudale d'ara rouge sur les danseurs, leurs clarinettes et le public. Il existe, chez les Wayãpi, un lien symbolique très fort associant la figure de l'anaconda, la racine de yamalatay et la plume caudale de l'ara rouge. L'association entre la danse et la plante est particulièrement significative puisque la plante est aussi nommée yalimã parce qu'elle "se tord comme l'anaconda" (ibid.: 184); mais le mot signifie également "faire un cercle » (Grenand 1989 : 134) ce qui renvoie à la façon de danser des Wayãpi. 
Photo 4. Pied de Zingiber zerumbet en forêt secondaire

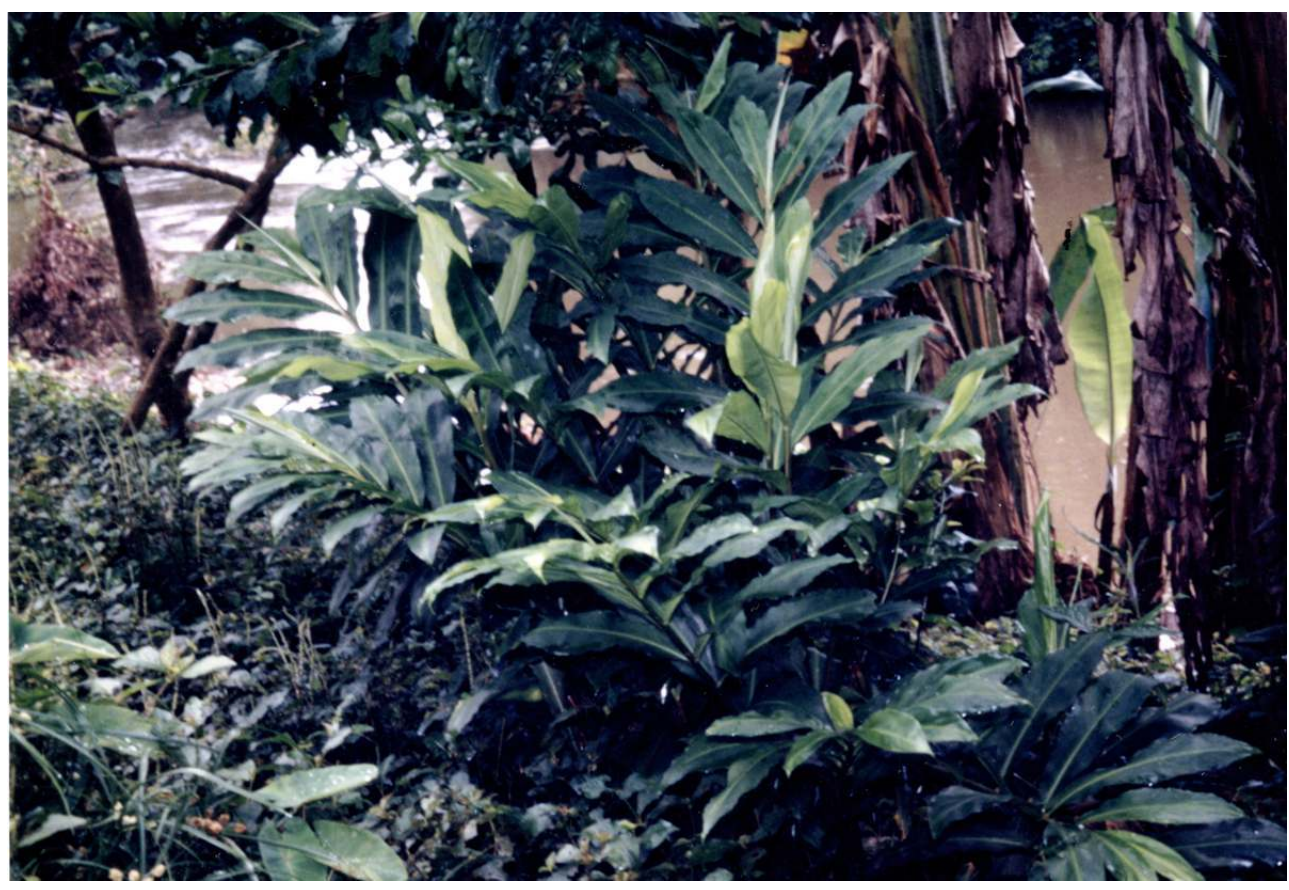

Cliché F. Grenand

67 Il est aussi très important de noter que les Wayãpi considèrent cette danse comme très ancienne et qu'ils la pratiquaient déjà avant leur migration depuis le sud de l'Amazone ; on peut y voir pour preuve qu'elle est connue de tous les sous-groupes wayãpi et comporte trente pièces distinctes (Beaudet 1997:63, 66). C'est donc probablement au contact des Portugais à la fin du XVII ${ }^{\mathrm{e}}$ siècle que l'emprunt de la plante - mais en aucun cas de ses usages - a dû se produire. 
Photo 5. Danse des grands poissons chez les Wayãpi de Trois Sauts : c'est sur des clarinettes TULE comme celles-ci qu'est frottée la macération de Zingiber zerumbet lors de la danse de l'anaconda

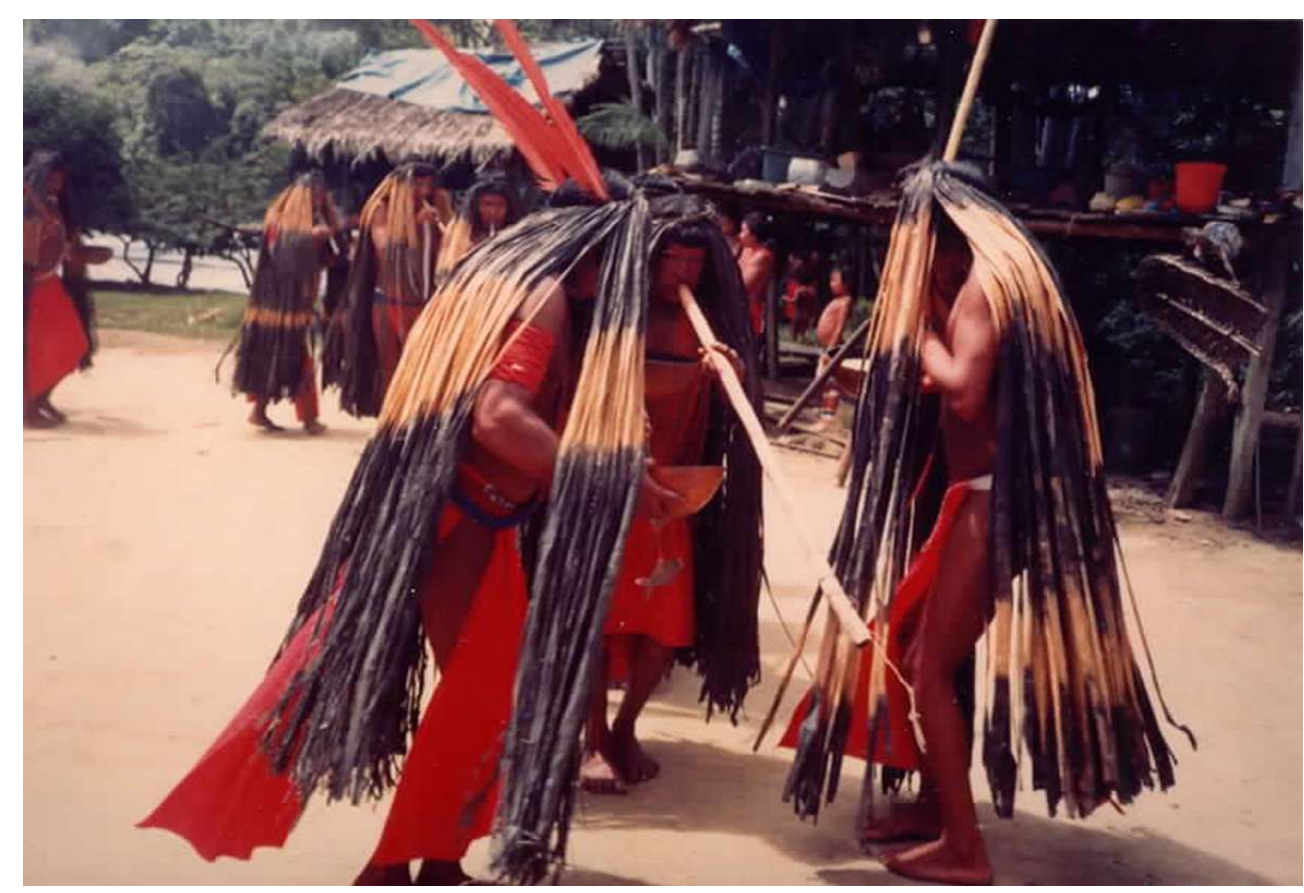

Cliché F. Grenand

La décoction des racines est par ailleurs utilisée en bain contre les atteintes malveillantes de l'anaconda (caractérisées par des convulsions) de façon identique à Rhipsalis baccifera (Cactacées). L'utilisation de ce remède est profane et, de ce fait, non contrôlée par les chamanes. La racine grattée et préparée en décoction courte est utilisée, toujours par les Wayãpi, comme carminatif pour soigner les coliques, ce qui constitue le seul lien avec les usages dans l'Ancien Monde.

Chez les Créoles, elle est utilisée comme Curcuma longa, c'est-à-dire comme vulnéraire pour soigner les foulures ou les coups : le rhizome est écrasé avec du sel, des feuilles de Siparuna guianensis (Monimiacées) et de Justicia pectoralis (Acanthacées), et mouillé de rhum, l'ensemble étant appliqué en cataplasme. Dans les cas de «coups reçus dans les côtes » (qui relève de la notion de blesse ${ }^{8}$ ), on prépare une décoction salée de ces trois plantes ; avant d'en boire trois fois par jour, on l'additionne de trois gouttes de teinture d'arnica et d'un peu de sucre.

Chez les Aluku, le rhizome de Zingiber zerumbet est utilisé de la même manière que le gingembre en infusion chaude contre les refroidissements ; contre la grippe, on peut aussi faire bouillir les feuilles dans l'eau, puis réduire en sirop en ajoutant du sucre; on y ajoute parfois d'autres plantes. Le rhizome entre également dans la composition de remèdes magiques (obia).

71 Les femmes utilisent les feuilles en bain de siège après les règles. On les utilise également en bain contre les mauvais esprits ainsi que pour laver les chiens de chasse (Fleury 1991, Fleury \& Topo, à paraître).

Les Wayana appellent la plante "ekei imë epit», ce qui signifie "le remède de l'anaconda "; en effet, selon les Wayana, le simple fait de voir un anaconda peut rendre malade, empêcher de dormir la nuit, ou donner de la fièvre. Les bains pris avec cette 
plante sont employés pour soigner ces malaises et maladies envoyés par l'anaconda. On répand aussi la plante sur le chemin pour éloigner l'animal.

Chez les Palikur, son utilisation est identique à celle de Zingiber officinale. Les racines pilées, appliquées en emplâtre, constituent un remède rafraîchissant contre les céphalées et les rhumatismes. Bues en tisane, elles sont aphrodisiaques et soulagent les maux de gorge (Berton 1997). Les feuilles bouillies avec des piments servent à préparer une potion bue pour soigner la tension. Ces utilisations sont toutes issues de la médecine créole.

Enfin chez les Kali'na qui connaissent un usage identique pour les deux Zingiber, les rhizomes, mis à macérer dans l'eau froide, servent à laver la tête des enfants malades dans le traitement de la fièvre et, selon Ahlbrinck ([1931] 1956 : 433), à soigner les maux de gorge.

L'examen détaillé des usages médicinaux souvent conjoints des deux Zingiber tend à montrer que la dominance passée de Zingiber officinale est probablement illusoire. On soulignera également la richesse des usages en Guyane française faisant écho aux observations de Barrère au XVIII ${ }^{\mathrm{e}}$ siècle.

\section{Du côté des noms populaires}

Nous nous limiterons ici à l'examen de quelques noms populaires propres à l'Amérique tropicale montrant que la création ou le voyage des mots (Grenand 1995) ne suit pas forcément le voyage des espèces et parfois même nous induit en erreur, comme le prouve la dénomination d'" hibiscus des Antilles " pour Abelmoschus moschatus, parfois appelée aussi « herbe à la poudre de Chypre » (Dictionnaire de Littré).

77 Cependant les noms les plus communément employés pour cette espèce sont souvent des composés du nom vernaculaire du gombo (A. esculentus) sous sa forme africaine la plus connue, okra ou encore sa forme caribéenne calou. Souvent ces composés font allusion à l'association de l'espèce avec les forces magiques telles que pisana ${ }^{9}$ en wayãpi, calou-diable [kalou-djab] en créole, wahitye akawa en palikur ou jorka-okro en sranan tongo soit, dans les trois derniers cas, "gombo des esprits». L'évocation de ce pouvoir magique est certainement à mettre en lien avec l'odeur caractéristique des graines. Les autres noms, ambrette, almizclillo ... renvoient par ailleurs essentiellement au parfum musqué ou ambré des graines (cf. Annexe).

Les noms les plus communément employés pour Zingiber officinale et Zingiber zerumbet sont majoritairement le mot "gingembre » (espagnol jenjibre, portugais gengibre, anglais ginger, aluku djinja et gaan djindja, kali'na sisibele...) et ses composés.

Néanmoins, un groupe de mots couvrant à la fois $Z$. officinale et $Z$. zerumbet mérite notre attention. Il s'agit du groupe construit au Brésil autour de mangará. Ce terme désigne au $\mathrm{xx}^{\mathrm{e}}$ siècle les bractées du bananier formant cône avant la floraison et la fructification (Matta 1938: 211; Buarque de Holanda Ferreira 1975: 878; Gregório 1987: 889-90). Cependant, selon Cunha (1982 : 202-203), il s'agit d'un sens récent, le mot s'appliquant du $\mathrm{XVI}^{\mathrm{e}}$ au $\mathrm{XIX}^{\mathrm{e}}$ siècle à des Aracées aux tubercules comestibles, sans aucun doute des Xanthosoma d'après la description précise de Soares de Sousa ([1587] 1971: 329). Selon Gregório (1987 : 889), ce nom s'applique aussi aux Caladium (autres Aracées) en synonymie de tajá. Il existe aussi des composés comme mangarito (ou mangará-mirim), litt. " petit mangará ", désignant l'Aracée Xanthosoma violacea et surtout mangarataia, Curcuma longa selon Buarque de Holanda Ferreira (1975: 878). Au moins au début du xxe siècle, ce 
dernier terme, qui signifie «mangará pimenté", s'appliquait en Amazonie à Z. officinale selon Corrêa ([1926] 1984: 383), ce que confirme Gregório (1987: 890). Quant à Matta (1938: 211) il indique que le terme mangarataia signifie "racine pimentée", qu'il est synonyme de gingembre et qu'il recouvre des Zingibéracées «com as variedades de rhyzomas amarello e branco ». Cette remarque induit très probablement l'existence en Amazonie brésilienne des deux espèces. On retrouve aussi ce terme sous la forme mankarata en quechua de Bolivie pour Z. officinale (Girault 1984: 152). Enfin, on peut rattacher à cet ensemble le mot yamalatay des Wayãpi pour Z. zerumbet auquel ils ne donnent aucune signification particulière, ainsi que marakatai en tembé et en urubukaapor, selon Balée (1994: 309), pour Z. officinale qui est considéré comme non décomposable. Cet imbroglio sémantique a, en définitive, le mérite de nous montrer que les gingembres ont basculé depuis longtemps dans les systèmes cognitifs indigènes.

\section{Conclusion : pour une étude de la migration des plantes secondaires}

80 Le cheminement qui précède montre que l'histoire naturelle des plantes d'intérêt économique secondaire permet de documenter deux approches contrastées :

- la diffusion des savoirs et leur transformation dans des contextes historiques et des environnements variés. L'exemple des Zingiber, dont la distinction des espèces n'est pas évidente, montre bien que leur cheminement n'est pas toujours facile à suivre. En revanche la présence d'Abelmoschus moschatus et de Z. zerumbet dans des populations plutôt isolées, montre que l'histoire de la diffusion de ce type de plante est tout aussi importante que celle des grandes espèces cultivées.

- l'appropriation culturelle (nomination nouvelle, usages nouveaux) par des cultures les plus diverses. Les processus de (re)nomination sont souvent des bons indicateurs d'appropriation. L'exemple du moypoã wayãpi ou de kapuwa oko des Aluku pour Abelmoschus moschatus sont autant de pistes suggérant un usage spécifique. Le tableau comparatif qui suit tend à prouver que cette appropriation est encore plus grande pour ce qui concerne les usages qui se diversifient, s'enrichissent ou s'appauvrissent selon les espèces et les sociétés.

Ce tableau permet aussi de mieux comprendre l'intrication tant de la diffusion des savoirs que de leur appropriation culturelle à travers soit des usages nouveaux, soit des usages lentement transformés. 
Tableau 1. Usages comparatifs dans l'Ancien Monde, l'Amérique et les Guyanes (en gras : usages spécifiques à l'Amérique tropicale et aux Guyanes)

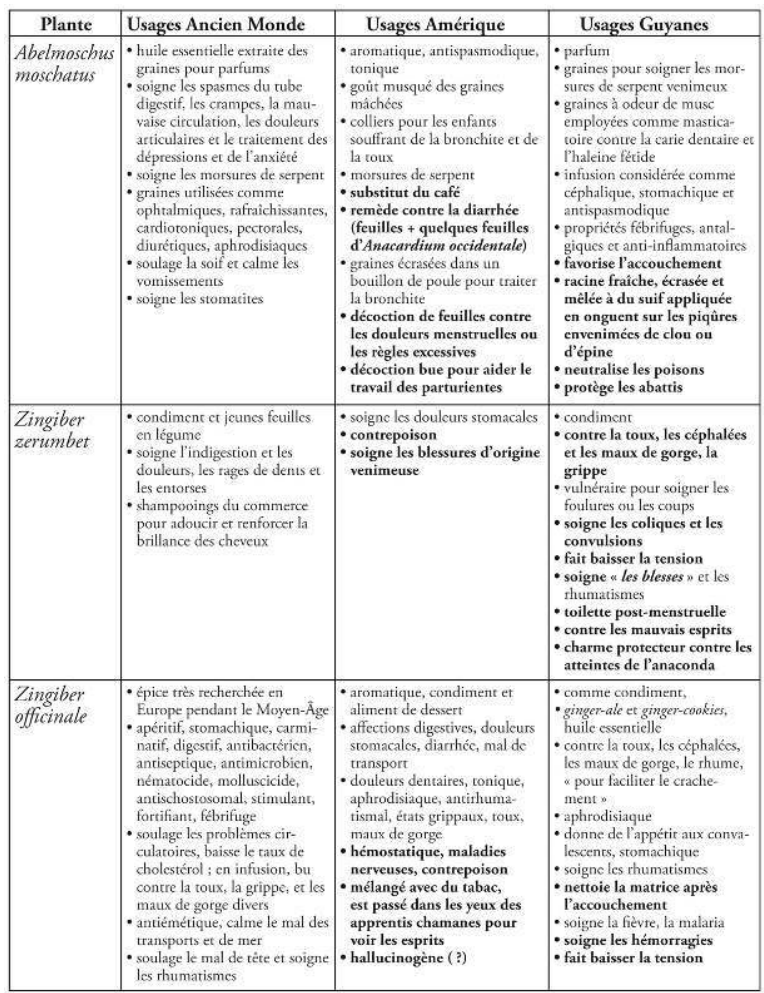

82 Nous espérons que notre démonstration incitera les anthropologues, les biologistes et les agronomes à mieux prendre en compte l'importance de la diffusion, par l'homme, de plantes économiquement secondaires ainsi que sa capacité d'innovation dans la préservation et l'amélioration de la biodiversité. Au final, il ne s'agit plus seulement de biodiversité mais bien de la richesse culturelle que les sociétés humaines réinventent sans cesse au cours de leur longue pérégrination planétaire.

\section{BIBLIOGRAPHIE}

Acevedo-Rodriguez P. \& Strong M.T. 2005 - Monocotyledons and Gymnosperms of Puerto Rico and the Virgin Islands. Washington DC, Department of Botany, National Museum of Natural History, $415 \mathrm{p}$. Agharkar S.P. 1991 - Medicinal plants of Bombay Presidency. Jodhpur, India, Scientific Publishers, 250 p. [First reprint, part 1-2].

Ahlbrinck W.G. 1931 - Encyclopaedie der Karaïben. Verhandelingen der Koninklijke Akademie van Wetenschappen, Amsterdam, 544 p. 1956, traduction française: L'Encyclopédie des Caraïbes, Paris, IGN.

Anonyme 1999 - Tacana : conozca nuestros árboles, nuestras hierbas. La Paz, FONAMA, IRD, UMSA, CIPTA, $497 \mathrm{p}$. 
Balée W. 1994 - Footprints of the Forest. Ka'apor Ethnobotany the Historical Ecology of Plant Utilization by an Amazonian People. New York, Columbia University Press, 396 p.

Barrère P. 1741 - Essai sur l'Histoire Naturelle de la France Equinoxiale. Paris, Piget, 215 p.

Bates D.M., 1968 - Notes on the Cultivated Malvaceae 2. Abelmoschus. Baileya 16: 99-112.

Beaudet J.-M. 1997 - Souffles d'Amazonie. Les orchestres tule des Wayãpi. Paris, Société d'ethnologie, CID Diffusion, 212 p. (Collection Hommes et musiques).

Bennett B.C. 1992 - Hallucinogenic Plants of the Shuar and Related Indigenous Groups in Amazonian Ecuador and Peru. Brittonia 44 (4): 483-493.

Bennett B.C. \& Prance G.T. 2000 - Introduced Plants in the Indigenous Pharmacopoeia of Northern South America. Economic Botany 54 (1) : 90-102.

Berton M.-E. 1997 - Les plantes médicinales chez les Amérindiens Palikurs de St Georges de l'Oyapock et Macouria (Guyane Française). DEA Environnement, Temps, Espaces, Sociétés, Universités de Paris VII/Orléans, $205 \mathrm{p}$.

Bown D. 1995 - Encyclopaedia of Herbs and their Uses. London, Dorling Kindersley, 424 p.

Buarque De Holanda Ferreira A. 1975 - Novo Dicionario da Lingua Portuguesa. Rio de Janeiro, Editora Nova Fronteira, 1499 p.

Burtt B.L. \& Smith R. M. 1983 - Zingiberaceae. In Dassanayake M.D. (Ed.) A Revised Handbook to the Flora of Ceylon, vol. IV, Smithsonian Institution, 488-532.

Corrêa M.P. [1926] 1984 - Dicionário das Plantas úteis do Brasil e das exóticas cultivadas. Rio de Janeiro, Imprensa Nacional Brasília, 6 vol.

Cunha A.G. da 1982 - Dicionário Histórico das Palavras Portuguesas de Origem Tupi. São Paulo, Melhoramentos, $357 \mathrm{p}$.

Duke J.A. \& Vasquez Martinez R. 1994 - Amazonian Ethnobotanical Dictionary. CRC Press, 215 p.

Duss R.P. [1897] 1972 - Flore phanérogamique des Antilles Françaises (Martinique et Guadeloupe, Heckel É. (collaborateur). Mâcon ,Protat Frères. Réédition Fort-de-France, Société de distribution et de culture, 2 vol., 656 p.

Fleury M. 1991 - Busi-nenge, les Hommes-Forêt. Essai d'ethnobotanique chez les Aluku (Boni) en Guyane française. Thèse de doctorat, Université Paris VI, 357 p.

Fleury M. \& Topo L. à paraître - Éléments de pharmacopée aluku.

Fournet J. 1978 - Flore illustrée des phanérogames de Guadeloupe et de Martinique. Paris, INRA, 2 vol. , 1054 p. ( $2^{\text {ème }}$ édition revue et corrigée, 2002, CIRAD, Montpellier, 2538 p.)

Fusée-Aublet J.B.C. 1775 - Histoire des Plantes de la Guiane Françoise. P.-F. Londres et Paris, Didot Jeune, 4 vol. (Reprint 1977, J. Cramer, Vaduz, Liechtenstein, 974 p.)

Fryxell P.A. 1992 - Malvaceae in Flora de Veracruz, fasc. 68. Xalapa, Veracruz, México, Instituto de Ecologiá, A.C., 255 p.

Girault L. 1984 - Kallawaya, Guérisseurs itinérants des Andes. Recherche sur les pratiques médicinales et magiques. Paris, Éditions de l'ORSTOM, 668 p. (Coll. Mémoires, 170).

Gregório I.J. 1987 - Contribuição Indígena ao Brasil. Belo Horizonte, União Brasileira de Educação e Ensino, 1316 p., 3 vol.

Grenand F. 1989 - Dictionnaire wayãpi-français. Lexique français-wayãpi (Guyane française). Paris, Peeters/SELAF, 538 p. (Coll. Langues et Sociétés d'Amérique Traditionnelle, 274). 
Grenand F. 1995 - Le voyage des mots. Logique de la nomination des plantes : exemples dans des langues tupi du Brésil. Les Cahiers du Lacito $7: 23-42$, Paris, Peeters. (Numéro thématique « Les mécanismes du changement culturel et linguistique »).

Grenand P., Moretti C., Jacquemin H. \& Prévost M.-F 2004 - Pharmacopées traditionnelles en Guyane : Créoles, Wayãpi, Palikur. Paris, IRD Éditions, 816 p. ( $2^{\text {ème }}$ édition entièrement revue et corrigée, 360 photographies en couleurs, 50 dessins au trait).

Grisebach A.H.R. [1864] 1963 - Flora of the British West Indian Islands. New York, Reprint by J. Cramer, 789 p.

Heckel É. 1897 - Les Plantes médicinales et toxiques de la Guyane Française. Mâcon, Protat Frères impr., 149 p.

Lemée A. 1956 - Flore de la Guyane Française. Tome 4. Paris, Éditions Paul Lechevalier, 133 p.

Lindley J. [1838] 1985 - Flora medica. New-Delhi, Ajay Book Service.

Longuefosse J.-L. 1995 - 100 plantes médicinales de la Caraibe. La Trinité, Gondwana Éditions, 238 p.

Maas P. J.M. 1985 - Zingiberaceae. In Gôrts-Van-Rijn A.R.A. \& Maas P.J.M.(Eds), Flora of the Guianas. Series A: Phanerogams. 192. Musaceae, 193. Zingiberaceae, 195. Cannaceae. Königstein, Koeltz Scientific Books : 29-67.

Marcgrave J. 1942 - História Natural do Brasil. (Trad. Magalhães J.P. de) São Paulo, Impresa Oficial do Estado, 293 p. (1 ère édititon 1648, Piso G \& Marcgrave G. Historia Naturalis Brasiliae).

Matta A.A. da 1938 - Contribuição ao Estudo do Vocabulario amazonense. Revista do Instituto de Geografia e História do Amazonas 6 (1-2): 21-363.

Ostendorf F.W. 1962 - Nuttige planten en sierplanten in Suriname. Landbouwproefstation in Suriname. Bulletin (79), Amsterdam, Koninklijk Instituut voor de Tropen, 325 p.

Rosengarten F. 1969 - The Book of Spices. Wynnewood, Pennsylvania, Livingston Publishing Co., $489 \mathrm{p}$.

Schultes R.E. \& Raffauf R.F. 1990 - The Healing Forest: Medicinal and Toxic Plants of the Northwest Amazonia. Portland, Dioscorides Press, 484 p.

Simmonds N.W. (Ed.) 1976 - Evolution of Crop Plants. London and New York, Longman, 339 p.

Soares de Sousa G. [1587] 1971 - Tratado descritivo do Brasil em 1587. Companhia Editora Nacional, São Paulo, Editora da universidade de São Paulo, 389 p. (Coll. Brasiliana, 117).

Van Andel T.R. 2000 - Non-timber forest products of the North-West District of Guyana. Georgetown, Guyana, Tropenbos Guyana, Part I: 320 p., Part II: 341 p. (Tropenbos Guyana Series 8A).

Walter A. \& Lebot V. 2003 - Jardins d'Océanie. CIRAD, Montpellier, 325 p.

\section{ANNEXES}

\section{Synonymes}

Abelmoschus moschatus Medik.= Hibiscus abelmoschus L., Hibiscus moschatus (Medik.) Salisb., Hibiscus abelmoschatus (L.) Karsten, Hibiscus collinsianus Nutt. ex Tor. \& A.Gray, Bamia abelmoschus R.Br. ex Wall.

Zingiber zerumbet (L.) Sm.= Amomum zerumbet L., Costus glabratus Sw. 
Zingiber officinale Roscoe= Amomum zingiber L., Zingiber zingiber H.Karst., Z. majus Rumph., Z. aromaticum Noronha, Zingiber missionis Wall.

\section{Noms vernaculaires}

Abelmoschus : de l'arabe halb el-mosk, " graine de musc », parce que les graines ont une odeur de musc.

Abelmoschus moschatus

Anglais : musk okra

Brésil : ambreta, malva algodão, almiscar, quigombó de cheiro

Colombie : ojo de gato

Equateur : anya-nye-nye (siona de Shushufindi)

Français : ambrette, graine musquée, hibiscus des Antilles, herbe à la poudre de Chypre. Guadeloupe et Martinique : gombo musqué (créole)

Guyane française : calou-diable[kalou-djab], calou grand bois[kalou-gran-bwa], calou sauvage (créole), moypoã, pisana (wayãpi), wahitye akawa (palikur), ëkëi epit (wayana), kapuwa oko (aluku).

Guyana : snake scent

Inde : muskdana

Pérou : almizclillo, ta-ta-amo (tikuna)

Surinam : wilde oker (néerlandais du Surinam), jorka-okro (sranan)

Zingiber zerumbet

Anglais : shampoo ginger

Brésil : gengibre amargo

Ceylan : wal-inguru, arankaha

Guyane française : gingembre, chichima (créole), yamalatay, moyupoã (wayãpi), gaan djindja (aluku), sisibele (kali'na), ëkëiimë epit (wayana)

Guyana : wild ginger

Hawaï : awapuhi

Poto-Rico : jenjibre amargo

Surinam : lempoejang (javanais)

Vanuatu : zerumbet, gingembre blanc

Zingiber officinale

Anglais : ginger

Français : gingembre

Bolivie : mankarata (quechua), shaute uja (tacana)

Brésil : gengibre, mangarataia, pia-du-udi (tukano), marakatai (urubu-kaapor, tembé)

Ceylan : inguru

Colombie : ajijilla

Equateur : jenjibre, ajilla, pia-nuni (secoya), afifindi (kofán) ajej (achuar)

Guadeloupe et Martinique : gingembre

Guyana : ginger

Guyane française : isuu (palikur), gingembre [jinjemb] (créole), djindja (aluku), walima (wayana)

Inde : adrak (hindi) 
Surinam : gember (Sranan), djahé (javanais), sisiperï, sinsipere (kali'na), shishimbiri (arawak) Pérou :jenjibre, kión

Vanuatu : gingembre commun

\section{Échantillons de référence}

Abelmoschus moschatus : Berton 127, Fleury 103, Fleury 977, Grenand 644, Jacquemin 1531, Jacquemin 1651, Jacquemin 2231, Jacquemin 2539, Prévost 1372, Sauvain 761.

Zingiber officinale: Berton 80, Fleury, 149, Fleury 314, Fleury 1461, Prévost 1369.

Zingiber zerumbet: Fleury 651, Fleury 722, Fleury 1263, Grenand 212, Grenand 503, Oldeman \& Burgot 960, Prévost 3956.

\section{NOTES}

1. Considéré comme wayãpi par nos informateurs et identifié par des fragments de poterie autochtone, des instruments en fer (canon de fusil, sabre de marine...). D'après sa position géographique, il s'agit probablement de l'ancien village du chef José-Antonio, guide des voyageurs De Bauve et Ferré en 1830.

2. Procédé d'extraction: extraction sélective au $\mathrm{CO}_{2}$ supercritique (cf. http://www.aromazone.com/aroma/extraits_Co2.asp).

3. http://sweetgum.nybg.org/vh/specimen_list.php, site du New York Botanical Garden.

4. http://mobot.mobot.org/cgi-bin/search_vast / http://www.tropicos.org/namesearch.aspx

5. Le commentateur moderne de la partie botanique de l'ouvrage, A.J. de Sampaio, indique cependant (p. XL, note 114) qu'il s'agit probablement d'un Althea, ce qui nous semble tout à fait erroné.

6. En Guyane, Zingiber zerumbet se maintient bien en forêt secondaire, ce qui lui confère une apparence de plante spontanée.

7. Les tule sont des clarinettes en bambou auxquelles sont associées des danses extrêmement variées. L'extension géographique de ces instruments est pan-amazonienne.

8. Nous avons traité de ce syndrome, propre aux médecines créoles, dans Grenand et al. 2004.

9. Ce mot est un emprunt, par les Wayãpi du sud, du mot pusanga propre à la Língua Geral et qui désigne l'ensemble des plantes magiques.

\section{RÉSUMÉS}

La diffusion des espèces végétales économiquement secondaires ne retient guère actuellement l'attention des ethnobotanistes. Les deux plantes que nous avons choisies, Abelmoschus moschatus et Zingiber zerumbet présentent des contrastes marqués. La première, largement diffusée, a été intégrée par les populations d'Amazonie avec une bonne partie de ses usages d'origine, alors que la seconde, d'usage plus localisé, a connu des innovations importantes. C'est en partant du prétexte des circonstances de leur «redécouverte » chez les Wayãpi que nous retraçons leur « histoire naturelle ». 
The dissemination of economically secondary plant species currently barely catches the attention of ethnobotanists. The two plants we selected, Abelmoschus moschatus and Zingiber zerumbet, present marked contrasts. The first, widely disseminated, was integrated by Amazonian populations together with most of its original uses, whereas the second, with more localised uses, underwent important innovations. Based on the pretext of the circumstances of their "rediscovery" by the Wayãpi Indians, we recount their "natural history".

\section{INDEX}

Mots-clés : ethnobotanique, migration des plantes, dynamique des usages

Keywords : ethnobotany, migration of plants, dynamics of uses

Population Wayãpi

\section{AUTEURS}

\section{PIERRE GRENAND}

IRD, Directeur de Recherche Émérite

Centre IRD de Guyane, BP 165, 97323 Cayenne

pgrenand@yahoo.fr

\section{MARIE-FRANÇOISE PRÉVOST}

IRD, AMAP/UMR 123

Centre IRD de Guyane, BP 165, 97323 Cayenne

marie-francoise.prevost@ird.fr

\section{MARIE FLEURY}

UMR 208 Patrimoines locaux, MNHN/IRD

Centre IRD de Guyane, Antenne Muséum, BP 165, 97323 Cayenne

fleury@mnhn.fr 